\title{
Discussion Summaries
}

Functional Dyspepsia and Gastroparesis: A spectrum of the same disease?

John P. Haydek, MD'; Zubair Malik, MD²; Madhusudan Grover, MBBS³

${ }^{1}$ Division of Gastroenterology and Hepatology, University of Colorado, Aurora, CO

${ }^{2}$ Section of Gastroenterology and Hepatology, Temple University, Philadelphia, PA

${ }^{3}$ Division of Gastroenterology and Hepatology, Mayo Clinic, Rochester, MN

\section{Corresponding Author}

John Haydek, MD

$12631 \mathrm{E} 17^{\text {th }}$ Ave.

MS B-158

Aurora, CO 80045

John.haydek@CUAnschutz.edu

Keywords: gastroparesis, functional dyspepsia, gastric emptying, gastric biopsy, interstitial cells of Cajal

Financial Disclosures \& Conflicts of Interest: The authors report no conflicts of interest. 


\title{
Discussion Summaries
}

\begin{abstract}
We summarize the @GIJournal discussion held on May 19, 2021 that covered the manuscript by Pasricha et al., titled "Functional Dyspepsia and Gastroparesis in Tertiary Care are Interchangeable Syndromes with Common Clinical and Pathologic Features." During the discussion, our expert Dr. Madhusudan Grover (MG) reviewed key findings and Dr. Zubair Malik (ZM) moderated the session.
\end{abstract}

\section{Introduction}

Chronic nausea and vomiting, early satiety and post-prandial fullness are common and affect up to $30 \%$ of the population (1). After presenting with such symptoms, patients are often evaluated using gastric emptying scintigraphy (GES) and diagnosed with gastroparesis (GP) if gastric emptying is delayed, or with functional dyspepsia (FD) if gastric emptying is normal. Until recently, clinical management of each condition has been different, although some gastroenterology societies have begun recommending against the use of GES and for similar treatment regardless of the GES results (2).

In the recent article, "Functional Dyspepsia and Gastroparesis in Tertiary Care are Interchangeable Syndromes with Common Clinical and Pathologic Features" by Pasricha et al. (3), a prospective, multi-site observational study was performed to characterize the symptom profile and clinical course of GP and FD and whether GES results remained stable over time. Additionally, enteric neuropathologic changes were compared between a subset of patients who underwent full-thickness gastric biopsy.

In the 944 patients included in the study, 720 (76\%) were diagnosed with GP and 224 (24\%) with FD. Both groups were predominantly white ( $89 \%$ for both groups) and female ( $84 \%$ of GP, $89 \%$ of FD). On enrollment, the GP group reported more severe symptoms, as measured by the Patient Assessment of Upper Gastrointestinal Disorders Symptom Severity Index and the Gastroparesis Cardinal Symptom Index. Additionally, the GP group had higher use of PPIs (75\% vs. $67 \%$ ), prokinetics ( $46 \%$ vs. $29 \%$ ) and total parental nutrition ( $7 \%$ vs. $3 \%$ ) at time of enrollment. After 48 weeks, patients from both groups were reassessed. There were no significant differences in change in symptoms between the groups. Repeat GES was performed, with $26 \%$ of the GP group and $27 \%$ participating. Based on the results of the repeat GES, $41 \%$ of patients had their initial diagnosed changed, with $42 \%$ of GP patients having a normal GES, and $37 \%$ of FD patients having a delayed GES. Additionally, 9 patients from each cohort underwent full-thickness gastric biopsies and results were compared to 9 patients from a control group. Within both the GP and FD groups, significant decreases in interstitial cells of Cajal (ICC) and anti-inflammatory myenteric macrophages were seen compared to healthy controls.

Based on the results, the GP and FD groups were unable to be differentiated at 48 weeks using GES. Furthermore, the same pathology was seen on full-thickness gastric biopsies in both groups. These findings together suggest that these two diagnoses may be inconstant and may more accurately reflect different phenotypes of a common underlying disease state. 


\section{Discussion Summaries}

\section{Discussion \\ @JHaydek: The big questions I took away from this study: Are GP and FD the same disease? If no, is it worth distinguishing between them? Should we treat both with the same therapies?}

MG: We need to take a step back and realize that about 15 years ago, GP was as much of a black box as FD. Then, due to work by the GP consortium and others and available animal models of delayed GE, we learned a lot about GP and the effects of diabetes. Now, we have started with FD and there will be more of an emphasis moving forward. Our thought is that they belong of a spectrum.

@OGrady_Greg: What role do you think patient selection played in the results in this excellent study? Are patients with severe disease visiting expert centers comparable to common FD in the wider community? Are they all the same spectrum?

MG: Great question. Certainly, these were tertiary care patients- more so, those undergoing biopsy. In fact, one of the reviewers wanted to see "tertiary" in title, for good reasons. I would like to do a longitudinal study in community practice patients.

MG: The future of diagnostics for "gastric neuromuscular disorders" is dynamic gastric emptying studies, MR spectrography, multi-sensor high resolution manometry and funcional luminal imaging panometry to evaluate antro-pyloric function.

@SalihSamo: Do you use the $8 \mathrm{~cm}$ EndoFLIP catheter for the pylorus? Is it challenging to place it across the pylorus? I do POEM and GPOEM and I plan to start using EndoFLIP for my GPOEM. I already use it for POEM.

ZM: For the pylorus, an easy way to do FLIP is to use a single channel therapeutic scope, as the probe will pass through the channel and you can advance it into the duodenum that way. There is a study by myself and Henry Parkman from a few years ago on this (4).

@SalihSamo: I assume you deflate the stomach all the way. Otherwise, you may get higher pressure reads that would affect your DI number. That shouldn't affect diameter/CSA.

ZM: Yes, I try to, although it's difficult if you have the catheter via the channel.

@LindaNguyenMD: Indeed, interpretation of this study has to be done with care as these are all patients seen at a tertiary referral center that specializes in GP and these patients typically have more severe symptoms. Also, the study design was designed to enrich for more patients with delayed gastric emptying. 


\section{Discussion Summaries}

MG: Yes, we stayed in that 4:1 to 5:1 ratio for GP vs FD. However, this may change in the future to have more FD.

@John_DamianosMD: Do you think in idiopathic GP that the GE abnormality (if there is one) is not the cause of symptoms (or the major cause) whereas in, say PD or others, it is?

@Bmoshiree: Yes, certainly different mechanism in patients with idiopathic disease. Remember though, Camilleri's study (5) showed that a well-done GES correlates with symptoms-but not in patients with diabetic GP.

\section{ZM: Should all patients with FD get a gastric emptying study?}

MG: I decide based on symptoms. If the patient has diabetes, I always do.

ZM: To further the discussion, should we still be getting a GES on patients with suspected GP? Or should we just treat symptoms?

@Bmoshiree: I generally order it. Can I guess results? Most of the time. Would I use a combination of medications with antiemetics and prokinetics? Probably. We have a tertiary practice though. For private folks, it may be more useful.

ZM: Based on the findings of decreased ICC in these patients and since it is seen in both, is there something we should be doing about this? Should we be placing stimulators in patients with FD?

@EsophagusDoc: That is an excellent question! Why were the patients classified as FD getting gastric stimulator in this study? That is how they got the full thickness biopsy. Is that why they look the same as GP group?

@Bmoshiree: I wondered the same! We don't do stimulators for FD but maybe they used to have delayed GES since most of those had idiopathic disease.

@LindaNguyenMD: Definitely not for pain. In GP, pain predominance was associated with nonresponse to Enterra [gastric neurostimulator]. Stimulators do not accelerate GE but likely modulate vagal afferent nerves in treating nausea and vomiting. So, if FD or chronic unexplained nausea and vomiting (CUNV) with more nausea and vomiting, it could be something to consider but we need more trials.

@LindaNguyenMD: These were likely "end stage" FD patients with nausea and vomiting predominance who previously responded to temporary stimulation. I don't know the details of the patients but knowing the authors, this is my hypothesis. 


\section{Discussion Summaries}

@EsophagusDoc: Great point @LindaNguyenMD! So, can we really say FD is the same as GP with pathophysiology based on looking at a handful of end stage FD and comparing to GP? That doesn't apply to majority of patients with FD.

MG: This is exactly why we are hoping to include wider spectrum of disease severity in our mechanistic studies. Both for GP and FD.

@JHaydek: As an aside, should we be placing stimulators in anyone?

MG: It could be considered in highly selected patients with vomiting predominance as an endof-the-line strategy. One could try temporary leads first to see if they respond. Dr. Tom Abell is a pioneer in that space.

@OGrady_Greg: Did you distinguish FD patients with or without CUNV overlap in this study? How is this relevant to the spectrum hypothesis?

MG: We don't distinguish FD from CUNV. They both have normal GE. On GPOEM, be careful. I have seen quite a few patients that are doing poorly due to rapid GE post GPOEM. Pyloric dysfunction is seen in only a subset of GP, so we need to be as selective as possible.

@EsophagusDoc: How do you select patients for GPOEM?

ZM: We tend to do a trial of either Botox or pyloric dilation first to see how a patient responds before going to GPOEM.

MG: Mayo and Hopkins will likely soon launch a clinical trial where GPOEM will be thoroughly assessed.

ZM: Why do you think that improvement was so poor in both groups of patients?

MG: Patients with severe disease. Plus, those who came back for a second GES were likely still suffering. It reflects that a lot of these patients continue to suffer and need more help from all of us and research. \#Unmetneeds

\section{Conclusion}

Chronic nausea and vomiting, early satiety and post-prandial fullness are common among patients and have traditionally been evaluated using GES and diagnosed as GP or FD based on the results. In this study, the GP and FD cohorts were unable to be differentiated at 48 weeks based on variable GES results or full-thickness gastric biopsies, when compared to controls. These findings call into question the use of GES to differentiate GP and FD, and in light of biopsies showing the same underlying pathophysiology, suggest that FD and GP may be 


\section{Discussion Summaries}

considered different presentations of the same underlying disorder. This reconceptualization of GP and FD to underlying gastric neuromuscular dysfunction due to a macrophage-driven Cajalopathy has significant management implications.

\section{References}

1. Stanghellini V, Chan FKL, Hasler WL, Malagelada JR, Suzuki H, Tack J, et al. Gastroduodenal Disorders. Gastroenterology. 2016;150(6):1380-92.

2. Moayyedi PM, Lacy BE, Andrews CN, Enns RA, Howden CW, Vakil N. ACG and CAG Clinical Guideline: Management of Dyspepsia. Official journal of the American College of Gastroenterology | ACG. 2017;112(7):988-1013.

3. Pasricha PJ, Grover M, Yates KP, Abell TL, Bernard CE, Koch KL, et al. Functional Dyspepsia and Gastroparesis in Tertiary Care are Interchangeable Syndromes With Common Clinical and Pathologic Features. Gastroenterology. 2021;160(6):2006-17.

4. Jehangir A, Malik Z, Petrov RV, Parkman HP. EndoFLIP and Pyloric Dilation for Gastroparesis Symptoms Refractory to Pyloromyotomy/Pyloroplasty. Digestive Diseases and Sciences. 2020.

5. Vijayvargiya P, Jameie-Oskooei S, Camilleri M, Chedid V, Erwin PJ, Murad MH. Association between delayed gastric emptying and upper gastrointestinal symptoms: a systematic review and meta-analysis. Gut. 2019;68(5):804-13. 